\title{
Spectrum of plasma modes and relevant transport processes in astrophysical disks
}

\begin{abstract}
B. Coppi
Massachusetts Institute of Technology, Cambridge, Massachusetts, USA

e-mail: coppi@mit.edu

Received 27 October 2008 / Accepted 29 June 2009

ABSTRACT

A simple plasma disk structure imbedded in a magnetic field and in the (prevalent) gravity of a central object is shown to be subject to the excitation of significant axisymmetric and tridimensional modes. The key factors involved in the relevant instability are the plasma pressure vertical gradient and the rotation frequency (around the central object) radial gradient. A modestly peaked vertical profile of the plasma temperature is shown to drive a "thermo-rotational instability" with considerable growth rates. Unstable modes are found as well for "flat" temperature profiles. The tri-dimensional tightly wound spirals that are found have properties that, unlike the familiar galactic spirals, depend on the vertical profiles of their amplitudes. Both radially standing and convective (quasi-modes) spirals are identified. Within the considered spectrum, unstable modes are shown to produce opposing fluxes of particles and thermal energies in the vertical direction. Thus disks with relatively flat vertical temperature profiles can expel particles (winds) from the equatorial plane while transporting thermal energy inward. An effective "diffusion" coefficient, for energy and angular momentum, is derived from the structure of radially convective spiral modes and shown to be consistent with significant radial transport rates.
\end{abstract}

Key words. accretion, accretion disks - black hole physics - magnetohydrodynamics (MHD) - instabilities - magnetic fields gravitation

\section{Introduction}

Identifying the plasma collective modes that can be excited in plasma disk structures (Pringle 1981; Blandford 1976; Lovelace 1976; Coppi \& Rousseau 2006) surrounding compact objects such as black holes can be important to explain experimental observations associated with objects of this kind. The geometry of these disk structures (Coppi \& Coppi 2001) and their physical parameters, that include in particular the radial gradient of the rotation frequency, the vertical gradients of the particle density and temperature and the effects of the magnetic field in which they are imbedded (Coppi 2008a), determine the characteristics and the spectrum of the modes that can be excited. Novel transport processes of particles and thermal energy in the vertical direction as well as radial transport of angular momentum can be produced by the identified modes. In fact, these processes and the tri-dimensional structure of the spiral modes (Coppi 2008b) that are found can be correlated with important observations connected with black holes (Coppi \& Rebusco 2008a). We point out that the properties of the tightly wound spirals that are introduced depend on the vertical profiles of their amplitudes, a feature that is not shared with the better known theory of galactic spirals (Bertin 2000). We note also that the theory presented here for both axisymmetric and tri-dimensional modes include, among others, the physical ingredients of basic modes such as the so called MRI instability (Velikhov 1959; Chandrasekhar 1960; Balbus \& Hawley 1991), that is appropriate for different geometries such as cylinders, or the modes that can be excited in well confined plasmas (Coppi \& Spight 1978) and can explain the experimentally observed inward transport of particles in connection with the outward transport of plasma thermal energy.
In Sect. 2 the characteristic parameters of the simplest plasma disk structure, imbedded in a vertical magnetic field and surrounding a compact object, are defined in view of the analyses presented in the next sections. In Sect. 3 the basic equations are given for the spectrum of axisymmetric and tri-dimensional normal modes that can be excited in a thin plasma disk. In Sect. 4 the realistic conditions on the density and temperature vertical profiles are examined under which nearly isobaric, weakly compressible modes are found. In Sect. 5 the theory of axisymmetric modes is given and the conditions for their marginal stability are derived. The radial gradient of the rotation frequency and the vertical gradient of the plasma pressure are recognized as the key factors for the excitation of these modes. In particular, when the ratio of the relative gradient of the plasma temperature exceeds the relative gradient of the density by $2 / 3$ a new form of instability (the "thermo-rotational instability") emerges (Coppi 2008a). This is in addition to that of the ballooning modes analyzed by Coppi \& Keyes (2003) that are found when the ratio of the gradients mentioned earlier is $2 / 3$ (polytropic profile). In Sect. 6 the transport produced in the vertical direction by the unstable modes described in Sect. 5 is evaluated by the relevant quasi-linear theory. Then the suggestion is made that the outward particle transport (away from the equatorial plane) occurring in the presence of relatively flat temperature profiles be the seed for the observed winds emanating from disks structures surrounding black holes (Elvis 2000). In this case an inward (toward the equatorial plane) flux of thermal energy is produced. The mode growth rates of the unstable modes on which the relevant transport coefficients depend are evaluated by completing the analysis given in Sect. 5. In Sect. 7 the characteristics of the weakly damped oscillatory modes, can be found when the 
temperature vertical profile is relatively flat, are examined. In Sect. 8 the theory of tri-dimensional spiral modes that are localized both in the vertical and the radial direction is given. These modes co-rotate with the plasma at the radius around which they are localized. The excitation of modes of this kind in the proximity of a black hole is, in fact, suitable to explain the observed high frequency quasi periodic oscillations (QPO's) as proposed by Coppi \& Rebusco (2008a). In Sect. 9 a new class of spiral quasi-modes that are convective in the radial direction and localized in the vertical direction are introduced. These modes are weakly damped and oscillatory in time and can be associated with the coupling to modes that are linearly unstable. An important property of the convective modes which are described is that they can transfer their angular momentum and energy at relatively fast rates in the radial direction. Thus, they may be considered as possible candidates to provide the plasma "viscosity" (Pringle 1981) associated with the mass accretion rates inferred from the luminosities of relevant objects. In Sects. 10 concluding remarks concerning the results presented in the previous sections are given.

\section{Simplest plasma structures}

The simplest configuration from which the modes that we shall analyze can emerge is a thin currentless disk that is threaded by a relatively weak vertical magnetic field $B_{z}$ and where the only component of the plasma flow velocity is toroidal. In particular, we assume that the central plasma pressure $p_{0}$ exceeds the magnetic pressure $B^{2} / 8 \pi$ The particle density profile for an up-down symmetric disk is represented by $n \simeq n_{0}\left(1-z^{2} / H_{0}^{2}\right)$ near the equatorial plane at the reference distance $R=R_{0}$ from the axis of symmetry. The thickness of the (density) disk, of the order $2 H_{0}$, is considered to be small relative to $R_{0}$. Different vertical temperature profiles, corresponding different heating processes, are represented near the equatorial plane by different values of the parameter

$$
\left.\eta_{T} \equiv-\frac{\mathrm{d} \ln T}{\mathrm{~d} z^{2}}\right)_{z=0} H_{0}^{2},
$$

where $2 T \equiv p / n=T_{\mathrm{e}}+T_{i}$ and $p$ is the total plasma pressure. The radial equilibrium equation, to lowest order in the ratio $H_{0}^{2} / R_{0}^{2}$, reduces to

$\Omega^{2}\left(R_{0}\right)=\frac{G M_{*}}{R_{0}^{3}} \equiv \Omega_{k}^{2}\left(R_{0}\right)$,

where $\Omega(R)$ is the rotation frequency, $\Omega_{k}\left(R_{0}\right)$ is the Keplerian frequency, $M_{*}$ is the mass of the central object and $v_{\phi}=\Omega R$ is the toroidal velocity. The relevant vertical equilibrium equation is

$0=-\frac{\partial p}{\partial z}-z \Omega_{k}^{2} \rho$

where $\rho$ is the mass density. Then Eq. (2) gives $4 T_{0}\left(1+\eta_{T}\right)=$ $H_{0}^{2} \Omega_{k}^{2} m_{i}$ for $z^{2} / H_{0}^{2} \ll 1$, where $m_{i}=\rho / n$. We shall consider a variety of temperature profiles including the flat profile corresponding to $\mathrm{d} T / \mathrm{d} z^{2}=0$ over the height of the disk $n=n_{0} \exp \left(-z^{2} / H_{0}^{2}\right)$ describes the entire density profile. The scale distance for the pressure gradient is defined by $H_{\mathrm{p}}^{2} \equiv$ $H_{0}^{2} /\left(1+\eta_{T}\right)$ for $z^{2} \ll H_{0}^{2}$.
The electric field that is present in the equilibrium configuration we have considered can be identified by referring to the electron momentum conservation equation

$-e n\left(\boldsymbol{E}+\frac{1}{c} \boldsymbol{u}_{\mathrm{e}} \times \boldsymbol{B}\right)-\nabla p_{\mathrm{e}} \simeq 0$

that gives

$E_{R}=-\Omega_{k}(R) \frac{R}{c} B_{z}$

and

$E_{z}=-\frac{1}{n e} \frac{\partial p_{\mathrm{e}}}{\partial z}$

where $p_{\mathrm{e}}$ is the electron pressure, $p=p_{\mathrm{e}}+p_{i}$ and $p_{\mathrm{e}} \simeq p_{i}$. Realistically, $\left|E_{z}\right| \ll\left|E_{R}\right|$.

Finally, we note that there can be different current carrying stationary plasma axisymmetric disk structures from that analyzed in this section. In particular, we may mention the configurations involving a sequence of plasma rings identified by Coppi $\&$ Rousseau (2006). We observe also that the modes analyzed in the next sections may be considered as "seeds" for new stationary configurations.

\section{Normal mode equations}

We consider normal mode perturbations from the indicated initial state represented by

$\hat{v}_{\phi}=\tilde{\tilde{v}}_{\phi}\left(R-R_{0}, z\right) \exp \left(\gamma_{0} t-\mathrm{i} \omega_{0} t+\mathrm{i} m_{\phi} \phi\right)$

in an interval $\left|R-R_{0}\right|$ around $R_{0}$, such that $\left|R-R_{0}\right| \ll R_{0}$, where $\gamma_{0}$ is the mode growth rate, $\omega_{0}$ the frequency, $m_{\phi}$ the toroidal mode number and $\hat{v}_{\phi}$ the perturbed toroidal velocity. The basic linearized equations that describe the departure from the initial state include

$\hat{\boldsymbol{E}}+\frac{1}{c}(\hat{\boldsymbol{v}} \times \boldsymbol{B}+\boldsymbol{v} \times \hat{\boldsymbol{B}})=0$,

$-\frac{1}{c} \frac{\partial \hat{\boldsymbol{B}}}{\partial t}=\nabla \times \hat{\boldsymbol{E}}$

and the total momentum conservation equation

$$
\begin{aligned}
\boldsymbol{A}_{m} \equiv & \rho\left(\frac{\partial}{\partial t} \hat{\boldsymbol{v}}+\hat{\boldsymbol{v}} \cdot \nabla \boldsymbol{v}+\boldsymbol{v} \cdot \nabla \hat{\boldsymbol{v}}\right) \\
& +\nabla\left(\hat{p}+\frac{\hat{\boldsymbol{B}} \cdot \boldsymbol{B}}{4 \pi}\right)-\frac{1}{4 \pi} \boldsymbol{B} \cdot \nabla \hat{\boldsymbol{B}}+z \hat{\rho} \Omega_{k}^{2} \boldsymbol{e}_{z}=0 .
\end{aligned}
$$

Here, we have taken into account that the initial magnetic field $B_{z}$ is considered to be varying over scale distances of the order of $R_{0}$ and have used standard symbols.

Furthermore, it is reasonable to assume that the collisional mean free path is short relative to the distance $\Delta_{z}$ over which the mode is localized vertically and to the mode radial wavelengths. Thus, the thermal conductivity can be neglected and the adiabatic equation of state can be adopted, that is,

$$
\frac{\partial}{\partial t} \hat{p}+\boldsymbol{v} \cdot \nabla \hat{p}+\hat{\boldsymbol{v}} \cdot \nabla p+\Gamma p \nabla \cdot \hat{\boldsymbol{v}}=0
$$


where $\Gamma=5 / 3$ is the adiabatic index. Then, given Eq. (6) we obtain

$\left[\gamma_{0}-\mathrm{i} \omega_{0}+\mathrm{i} m_{\phi} \Omega_{k}(R)\right] \hat{p}+\hat{v}_{z} \frac{\partial p}{\partial z}+\Gamma p \nabla \cdot \hat{\boldsymbol{v}}=0$

and we choose to consider modes that co-rotate with the plasma at $R=R_{0}$.

Therefore, we take

$\omega_{0}=m_{\phi} \Omega_{k}\left(R_{0}\right)+\delta \omega_{0}$,

where $\left|\delta \omega_{0}\right| \ll m_{\phi} \Omega_{k}\left(R_{0}\right)$ is not linear in $m_{\phi}$, and Eq. (6) reduces to

$\hat{v}_{\phi} \simeq \tilde{\tilde{v}}_{\phi}\left(R-R_{0}, z\right) \exp \left\{\gamma_{0} t-\mathrm{i} \delta \omega_{0} t+\mathrm{i} m_{\phi}\left[\phi-\Omega_{k}\left(R_{0}\right) t\right]\right\}$.

We define

$\gamma_{t} \equiv \gamma_{0}+\mathrm{i} \Omega_{k}^{\prime}\left(R-R_{0}\right)-\mathrm{i} \delta \omega_{0}, \quad \hat{\boldsymbol{v}}_{\mathrm{p}}=\gamma_{t} \hat{\boldsymbol{\xi}}_{\mathrm{p}}$

for $\hat{\boldsymbol{v}}_{\mathrm{p}}=\gamma_{t} \hat{\boldsymbol{\xi}}_{\mathrm{p}}$ where the subscript $p$ indicates the relevant poloidal component. Then, the perturbed pressure and density are given by

$\hat{p} \simeq-\hat{\xi}_{z} \frac{\mathrm{d} p}{\mathrm{~d} z}-\Gamma p \nabla \cdot \hat{\boldsymbol{\xi}}_{\mathrm{p}}$,

$\hat{\rho} \simeq-\hat{\xi}_{z} \frac{\mathrm{d} \rho}{\mathrm{d} z}-\rho \nabla \cdot \hat{\boldsymbol{\xi}}_{\mathrm{p}}$

as

$$
\begin{aligned}
\nabla \cdot \hat{\boldsymbol{v}} & \simeq \frac{\partial}{\partial R}\left(\gamma_{t} \hat{\xi}_{R}\right)+\gamma_{t} \frac{\partial}{\partial z} \hat{\xi}_{z}+\mathrm{i} m_{\phi} \frac{1}{R_{0}}\left(-\Omega_{k}^{\prime} R_{0} \hat{\xi}_{R}+\gamma_{t} \hat{\xi}_{\phi}\right) \\
& \simeq \gamma_{t}\left(\frac{\partial \hat{\xi}_{R}}{\partial R}+\frac{\partial}{\partial z} \hat{\xi}_{z}+\mathrm{i} \frac{m_{\phi}}{R_{0}} \hat{\xi}_{\phi}\right),
\end{aligned}
$$

and we consider $\left|m_{\phi} \hat{\xi}_{\phi} / R_{0}\right| \ll\left|\partial \hat{\xi}_{R} / \partial R\right|$.

The perturbed magnetic field components are derived from the combination of Eqs. (7) and (8) that gives

$\hat{B}_{\phi}=B_{z} \frac{\partial}{\partial z} \hat{v}_{\phi}+\frac{\mathrm{d} \Omega}{\mathrm{d} R} \hat{B}_{R}$

$\hat{B}_{R} \simeq B_{z} \frac{\partial}{\partial z} \hat{\xi}_{R}$

$\hat{B}_{z} \simeq B_{z}\left(\frac{\partial}{\partial z} \hat{\xi}_{z}-\nabla \cdot \hat{\xi}_{\mathrm{p}}\right)$

Thus, for

$\hat{\mathrm{v}}_{\phi}=-R \Omega^{\prime} \hat{\xi}_{R}+\gamma_{t} \hat{\xi}_{\phi}$

we have

$\hat{B}_{\phi}=B_{z} \frac{\partial}{\partial z} \hat{\xi}_{\phi}$

\section{Quasi-isobaric, weakly compressible modes}

As we shall see, the considered limit $\beta \equiv 8 \pi p_{0} / B^{2}>1$ leads to find that $\Delta_{z}^{2}<H_{0}^{2}$ and in this case the modes of interest can be characterized as "quasi-isobaric" and "weakly compressible" in the sense that

$\left|\frac{\hat{p}}{p}\right|<\left|\frac{\hat{\rho}}{\rho}\right| \quad$ and $\quad\left|\nabla \cdot \hat{\boldsymbol{\xi}}_{\mathrm{p}}\right|<\left|\frac{\partial \hat{\xi}_{z}}{\partial z}\right|$

implying that

$\frac{\hat{T}}{T} \simeq-\frac{\hat{\rho}}{\rho}$

and

$\frac{\partial \hat{\xi}_{R}}{\partial R} \simeq-\frac{\partial \hat{\xi}_{z}}{\partial z}$

To prove this point we take the z-component of Eq. (9)

$\rho \gamma_{t}^{2} \hat{\xi}_{z}+z \hat{\rho} \Omega_{k}^{2}=-\frac{\partial}{\partial z} \hat{p}$

and consider the gravitational term $z \hat{\rho} \Omega_{k}^{2}$ as important in Eq. (22), that is

$|z \hat{\rho}| \Omega_{k}^{2} \sim\left|\frac{\partial \hat{p}}{\partial z}\right|$,

where $|\partial \hat{p} / \partial z| \sim\left|\hat{p} / \Delta_{z}\right|$. Then $\Delta_{z}^{2}|\hat{\rho}| \Omega_{k}^{2} \sim|\hat{p}|$ and since $\Omega_{k}^{2} \sim$ $(p / \rho) / H_{0}^{2}$ the ordering (23) implies that

$\frac{|\hat{p}|}{p} \sim \frac{|\hat{\rho}|}{\rho} \frac{\Delta_{z}^{2}}{H_{0}^{2}}$.

Here we limit our considerations to modes for which $\Delta_{z}^{2} / H_{0}^{2}<$ 1 , as we shall show that $\Delta_{z}^{2} / H_{0}^{2} \sim 1 / \beta^{1 / 2}$ and we restrict our analysis to $\beta>1$ for the sake of simplicity. Then we have

$$
\begin{aligned}
\nabla \cdot \hat{\boldsymbol{\xi}}_{\mathrm{p}} & \simeq-\frac{3}{5} \hat{\xi}_{z} \frac{1}{p} \frac{\mathrm{d} p}{\mathrm{~d} z}=-\frac{3}{5} \hat{\xi}_{z}\left(\frac{1}{\rho} \frac{\mathrm{d} \rho}{\mathrm{d} z}+\frac{1}{T} \frac{\mathrm{d} T}{\mathrm{~d} z}\right) \\
& \simeq \frac{6}{5} \frac{z}{H_{0}^{2}}\left(1+\eta_{T}\right) \hat{\xi}_{z} .
\end{aligned}
$$

implying that

$\left|\nabla \cdot \hat{\boldsymbol{\xi}}_{\mathrm{p}}\right| \sim \hat{\xi}_{z} \frac{\Delta_{z}}{H_{0}^{2}}$

and that Eq. (21) is valid for $\Delta_{z}^{2}<H_{0}^{2}$. Moreover

$\frac{\hat{\rho}}{\rho} \simeq \frac{z}{H_{0}^{2}} \hat{\xi}_{z} C_{0}$

for

$C_{0} \equiv \frac{6}{5}\left(\eta_{T}-\frac{2}{3}\right)$

and $\hat{p}$ is obtained as a function of $\hat{\xi}_{z}$ from Eq. (21) that becomes

$\rho\left(\gamma_{t}^{2}-\frac{2 z^{2} C_{0}}{H_{0}^{2}} \Omega_{k}^{2}\right) \hat{\xi}_{z} \simeq-\frac{\partial}{\partial z} \hat{p}$. 
We see that the sign of $C_{0}$ is important in relating that of $\hat{\rho}$ to $\hat{\xi}$. Finally, we can verify that, for $m_{\phi}<R_{0} / H_{0}$, the toroidal pressure gradient can be neglected in the $\phi$-component of Eq. (9). Then this reduces to

$\gamma_{t}\left(\gamma_{t} \hat{\xi}_{\phi}+2 \Omega_{k} \hat{\xi}_{R}\right) \simeq v_{\mathrm{A}}^{2} \frac{\partial^{2}}{\partial z^{2}} \hat{\xi}_{\phi}$

In fact, we limit our analysis to the case where

$\left|m_{\phi} \frac{\mathrm{d} \Omega}{\mathrm{d} R}\left(R-R_{0}\right)\right|<\left(\gamma_{0}^{2}+\delta \omega_{0}^{2}\right)^{1 / 2}$.

Finally, it is convenient to refer to the $\partial\left(\boldsymbol{e}_{\phi} \cdot \nabla \times \boldsymbol{A}_{m}\right) / \partial z=0$ equation, derived from Eq. (9), that is

$$
\begin{aligned}
& \frac{\partial^{2}}{\partial z^{2}}\left[\rho\left(\gamma_{t}^{2} \hat{\xi}_{R}-2 \Omega_{k} \hat{\mathbf{v}}_{\phi}\right)-\frac{B_{z}^{2}}{4 \pi} \frac{\partial^{2}}{\partial z^{2}} \hat{\xi}_{R}\right] \\
& -\frac{\partial}{\partial z}\left\{\frac{\partial}{\partial R}\left[\rho \gamma_{t}^{2} \hat{\xi}_{z}+z \Omega_{k}^{2} \hat{\rho}+\frac{B_{z}^{2}}{4 \pi} \frac{\partial^{2}}{\partial R \partial z} \hat{\xi}_{R}\right]\right\}=0 .
\end{aligned}
$$

By using Eqs. (18) and (26) this can be rewritten as

$$
\begin{aligned}
& \frac{\partial^{2}}{\partial z^{2}}\left[\rho\left(\gamma_{t}^{2} \hat{\xi}_{R}+2 \Omega_{k} \Omega_{k}^{\prime}\right) \hat{\xi}_{R}-\frac{B_{z}^{2}}{4 \pi}\left(\frac{\partial^{2}}{\partial z^{2}}+\frac{\partial^{2}}{\partial R^{2}}\right) \hat{\xi}_{R}\right] \\
& -2 \Omega_{k} \gamma_{k} \frac{\partial^{2}}{\partial z^{2}} \hat{\xi}_{\phi}-\frac{\partial^{2}}{\partial z \partial R}\left[\rho\left(\gamma_{t}^{2}-\frac{z^{2}}{H_{0}^{2}} C_{0}\right) \hat{\xi}_{z}\right] .
\end{aligned}
$$

Thus we have a complete set of equation given by Eq. (30) combined with

$\frac{\partial \hat{\xi}_{z}}{\partial z} \simeq-\frac{\partial \hat{\xi}_{R}}{\partial R}$

and

$v_{\mathrm{A}}^{2} \frac{\partial^{2}}{\partial^{2}} \hat{\xi}_{\phi}-\gamma_{t}^{2} \hat{\xi}_{\phi} \simeq 2 \gamma_{t} \Omega_{k} \hat{\xi}_{R}$

that relate $\hat{\xi}_{z}$ and $\hat{\xi}_{\phi}$ to $\hat{\xi}_{R}$.

\section{Axisymmetric modes}

At first we consider, for simplicity, axisymmetric modes (Coppi 2008a) with $m_{\phi}=0$, that are purely growing. Thus, $\gamma_{t}=\gamma_{0}$ and we look for normal modes of the form

$\hat{\mathrm{v}}_{\phi} \simeq \tilde{\mathrm{v}}_{\phi}^{0} G_{0}(z) \exp \left[\gamma_{0} t+\mathrm{i} k_{R}\left(R-R_{0}\right)\right]$

that are a special case of those represented by Eq. (6) and where $k_{R}^{2} R_{0}^{2} \gg 1, k_{R} \sim k_{0} \equiv\left(-2 \Omega^{\prime} R \Omega\right)^{1 / 2} / v_{\mathrm{A}} \equiv 1 / L_{c}, \Omega^{\prime}=\mathrm{d} \Omega / \mathrm{d} R$, $\Omega_{k}^{\prime}=-3 \Omega_{k} /\left(2 R_{0}\right)$ and $v_{\mathrm{A}} \equiv B_{z} /(4 \pi \rho)^{1 / 2}$ is the Alfvén velocity. In addition $G_{0}(z)$ is an even or odd function of $z$ that is localized over a distance $L_{c}<\Delta_{z} \lesssim H_{\mathrm{p}}$ represented, for instance, by $G_{0}=$ $\exp \left[-z^{2} /\left(2 \Delta_{z}^{2}\right)\right]$. The unstable modes that are found have

$\Omega_{D}^{2} \equiv-2 \Omega_{k}^{\prime} R \Omega_{k}=3 \Omega_{k}^{2}>k_{R}^{2} v_{\mathrm{A}}^{2}$

corresponding to the fact that the radial gradient of the rotation frequency is a key driving factor for the relevant instability. Clearly, $k_{R}^{2} v_{\mathrm{A}}^{2}$ is the representative bending factor of the magnetic field lines.
Then Eq. (31) reduces simply to

$$
\begin{aligned}
& \frac{\partial^{2}}{\partial z^{2}}\left\{\left[\rho\left(\gamma_{0}^{2}-\Omega_{0}^{2}\right)+k_{R}^{2} \frac{B_{z}^{2}}{4 \pi}\right] \hat{\xi}_{R}-\frac{B_{z}^{2}}{4 \pi} \frac{\partial^{2}}{\partial z^{2}} \hat{\xi}_{R}\right\} \\
& -2 \Omega_{k} \gamma_{0} \rho_{0} \frac{\partial^{2}}{\partial z^{2}} \hat{\xi}_{\phi}-\mathrm{i} k_{R} \rho_{0} \frac{\partial}{\partial z}\left[\left(\gamma_{0}^{2}+\frac{z^{2}}{H_{0}^{2}} C_{0} \Omega_{k}^{2}\right) \tilde{\xi}_{z}\right] \simeq 0 .
\end{aligned}
$$

Here we consider $\gamma_{0}^{2} \sim\left(\Delta_{z}^{2} / H_{0}^{2}\right) \Omega_{k}^{2}<\Omega_{k}^{2}$ and therefore $k_{R}^{2} v_{\mathrm{A}}^{2} \simeq$ $\Omega_{0}^{2}\left(1-\epsilon_{k}\right)$ with $\epsilon_{k}<1$, that is $k_{R}^{2} \simeq k_{0}^{2}\left(1-\epsilon_{k}\right)$. Then since $\rho \simeq$ $\rho_{0}\left(1-z^{2} / H_{0}^{2}\right)$ and Eq. (29) becomes simplify

$\gamma_{0}^{2} \tilde{\xi}_{\phi}+2 \Omega_{k} \gamma_{0} \tilde{\xi}_{R}=v_{\mathrm{A}}^{2} \frac{\mathrm{d}^{2}}{\mathrm{~d} z^{2}} \tilde{\xi}_{\phi}$,

where $\tilde{\xi}_{R}=\tilde{\xi}_{R}(z)$ and $\tilde{\xi}_{\phi}=\tilde{\xi}_{\phi}(z)$ Eq. (34) can be reduced to

$$
\begin{aligned}
& \frac{\mathrm{d}^{2}}{\mathrm{~d} z^{2}}\left\{\Omega_{D}^{2}\left(1-\frac{k_{R}^{2}}{k_{0}^{2}}\right) \tilde{\xi}_{R}-\Omega_{D}^{2} \frac{z^{2}}{H_{0}^{2}} \tilde{\xi}_{R}+v_{\mathrm{A}}^{2} \frac{\mathrm{d}^{2}}{\mathrm{~d} z^{2}} \tilde{\xi}_{R}-\gamma_{0}^{2} \tilde{\xi}_{R}\right\} \simeq \\
& \frac{4}{3} k_{0}^{2} \gamma_{0}^{2}\left[\left(1+\frac{3}{4} \frac{k_{R}^{2}}{k_{0}^{2}}\right) \tilde{\xi}_{R}+\frac{\gamma_{0}}{2 \Omega_{k}} \tilde{\xi}_{\phi}\right]-\mathrm{i} k_{R} C_{0} \Omega_{k}^{2} \frac{\mathrm{d}}{\mathrm{d} z}\left[\frac{z^{2}}{H_{0}^{2}} \tilde{\xi}_{z}\right],
\end{aligned}
$$

where $\tilde{\xi}_{R} \simeq\left(i / k_{R}\right) \mathrm{d} \tilde{\xi}_{z} / \mathrm{d} z$. Since we look for localized solution soltuion in $z$ it is convenient to take the Fournier transform of Eqs. $\left(29^{\prime}\right)$ and (35) that gives

$\tilde{\xi}_{\phi k} \simeq-2 \Omega_{k} \gamma_{0} \frac{\tilde{\xi}_{R k}}{\gamma_{0}^{2}+k^{2} v_{\mathrm{A}}^{2}}$

where $k^{2} \sim 1 / \Delta_{z}^{2}$ and

$\mathrm{i} k_{R} \tilde{\xi}_{R k}+\mathrm{i} k \tilde{\xi}_{z k} \simeq 0$.

Then

$\tilde{\xi}_{\phi k} \simeq 2 \Omega_{k} \gamma_{0} \frac{k \tilde{\xi}_{z k}(k)}{k_{R}\left(\gamma_{0}^{2}+k^{2} v_{\mathrm{A}}^{2}\right)}$.

Moreover,

$\left\{\left(\frac{\gamma_{0} H_{0}}{v_{\mathrm{A}}}\right)^{2}\left(1+\frac{4}{3} \frac{k^{2} v_{\mathrm{A}}^{2}}{k^{2} v_{\mathrm{A}}^{2}+\gamma_{0}^{2}}\right)-k^{2}\left(\epsilon_{k}-\frac{k^{2}}{k_{0}^{2}}\right) H_{0}^{2}\right\} \tilde{\xi}_{z k}$

$\simeq k \frac{\mathrm{d}^{2}}{\mathrm{~d} k^{2}}\left(k \tilde{\xi}_{z k}\right)-\frac{C_{0}}{3} k_{0}^{2} \frac{\mathrm{d}^{2}}{\mathrm{~d} k^{2}} \tilde{\xi}_{z k}$,

and the problem is reduced to solving a second order differential equation.

We note that at marginal stability $\left(\gamma_{0}^{2}=0\right) \tilde{\xi}_{\phi k}$ and Eq. (39) reduces to

$0 \simeq\left(k H_{0}\right)^{2}\left(\epsilon_{k}-\frac{k^{2}}{k_{0}^{2}}\right) \tilde{\xi}_{z k}+k \frac{\mathrm{d}^{2}}{\mathrm{~d} k^{2}}\left(k \tilde{\xi}_{z k}\right)-\frac{C_{0}}{\mathrm{v}_{A}^{2}} \Omega_{k}^{2} \frac{\mathrm{d}^{2}}{\mathrm{~d} k^{2}} \tilde{\xi}_{z k}$.

Thus we obtain the known ballooning solution

$\tilde{\xi}_{z k}=\tilde{\xi}_{z k}^{\partial} \exp \left(-\frac{k^{2}}{2 \sigma}\right)$

where

$\sigma^{2}=\frac{k_{0}^{2}}{H_{0}^{2}} \equiv \frac{1}{\Delta_{0}^{4}}$ 
and

$E_{k}^{0} \equiv \epsilon_{k} k_{0} H_{0}=3$

for $C_{0}=0$. Clearly unstable modes of this kind can be found for $C_{0}<0$, that is for

$\eta_{T}>\frac{2}{3}$

and correspond to the "thermo rotational instability" presented in Coppi (2008a).

Since flat temperature profiles corresponding to $C_{0}<0$ are an important class. to be considered, higher modes, for which $E_{k}^{0}>3$, have to be analyzed. For these modes the influence of the radial gradient of the rotation frequency is stronger. Then it is convenient to use the variable $\bar{k} \equiv k \Delta_{0}$ and to rewrite Eq. (40) as

$\bar{k}^{2}\left(E_{k}^{0}-\bar{k}^{2}\right) \tilde{\xi}_{z k}+\bar{k} \frac{\mathrm{d}^{2}}{\mathrm{~d} \bar{k}^{2}}\left(\bar{k} \tilde{\xi}_{z k}\right)+C_{0}^{0} \frac{\mathrm{d}^{2}}{\mathrm{~d} \bar{k}^{2}} \tilde{\xi}_{z}=0$

where

$C_{0}^{0} \equiv C_{0}\left(\frac{k_{0} H_{0}}{3}\right)^{1 / 2}$

It is also useful to derive a quadratic form obtained by multiplying Eq. (40) by $\tilde{\xi}_{z k}$ and integrating it from $-\infty$ to $+\infty$. This gives

$E_{k}^{0}\left\langle\left(\bar{k} \tilde{\xi}_{z k}\right)^{2}\right\rangle=\left\langle\left(\bar{k}^{2} \tilde{\xi}_{z k}\right)^{2}\right\rangle+\left\langle\left[\frac{\mathrm{d}}{\mathrm{d} \bar{k}}\left(\bar{k} \tilde{\xi}_{z k}\right)\right]^{2}-C_{0}^{0}\left(\frac{\mathrm{d}}{\mathrm{d} \bar{k}} \tilde{\xi}_{z k}\right)^{2}\right\rangle$.

The next eigensolution is odd with

$\tilde{\xi}_{z k}=\tilde{\xi}_{z k}^{0} \bar{k} \exp \left(-\frac{\bar{k}^{2}}{2}\right)$.

In this case

$C_{0}^{0}=-\frac{2}{3}$

and

$E_{k}^{0}=\frac{13}{3}$.

Proceeding to the next eigenfunction we find

$\tilde{\xi}_{z k}=\tilde{\xi}_{z k}^{0}\left(1+\frac{1}{2} \bar{k}^{2}\right) \exp \left(-\frac{\bar{k}^{2}}{2}\right)$

and

$C_{0}^{0}=-\frac{14}{5}$

with

$E_{k}^{0}=\frac{21}{5}$.

For $C_{0}<-14 / 5$ a still higher eigensolution has to be considered.

\section{Vertical fluxes of particles and thermal energy}

The considered modes can produce particle density transport, in the vertical direction, that is of contrary sign to that of the temperature transport and modify the density and temperature profiles in such a way as to lead $\eta_{T}$ toward $2 / 3$, corresponding to a polytropic. Thus, if $\eta_{T}>2 / 3$ a particle inflow toward the equatorial plane is induced. When $\eta_{T}<2 / 3$, including the case where $\eta_{T}=0$ or where the surface of the disk can be hotter than the interior, the particle transport is away, from the equatorial plane. These arguments are based on the quasilinear analysis that gives the vertical particle flux produced by unstable modes as

$\Gamma_{p z}=\left\langle\left\langle\hat{n} \hat{v}_{z}\right\rangle\right\rangle \simeq-\frac{4}{5} \gamma_{0}\left\langle\left\langle\left|\hat{\xi}_{z}\right|^{2}\right\rangle\right\rangle \times\left[\frac{\partial}{\partial z} n-\frac{3}{2} \frac{n}{T} \frac{\partial}{\partial z} T\right]$,

where $\langle\langle\rangle\rangle$ indicates an average over a radial distance $\Delta R$ such that $1 / k_{R}<\Delta R<R_{0}$. The corresponding temperature flux is $\left\langle\left\langle\hat{T} \hat{v}_{z}\right\rangle\right\rangle \simeq-\left\langle\left\langle\hat{n} \hat{v}_{z}\right\rangle\right\rangle T / n$.

The outflows produced by these modes when $\eta_{T}<2 / 3$ can be considered as candidates to explain the origin of the particle fluxes (winds) that have been observed to emanate from disk structures such as those at the core of AGN's (Elvis 2000).

The transport process described by Eq. (51) is similar to that proposed for the theoretical explanation of the experimentally observed particle inflow in magnetically confined toroidal plasmas that is associated (Coppi \& Spight 1978) with the outflow of electron thermal energy related to the ratio of the gradients of the radial electron temperature and the particle density.

To illustrate this case we refer, for simplicity, to a cylindrical or plane plasma confinement configuration where the magnetic field is in the $z$ direction. Electrostatic modes can be excited with longitudinal phase velocities $\left|\omega / k_{\|}\right|<v_{\text {the }}, \mathrm{v}_{\text {the }}$ being the electron thermal velocity. Considering the limit where the electron longitudinal thermal conductivity is relatively large but finite, it is possible to show that the radial transport velocity for the electrons is equal to and of opposite sign of that of the electron thermal energy. In particular, when

$\eta_{\mathrm{e}} \equiv \frac{\mathrm{d} \ln T_{\mathrm{e}}}{\mathrm{d} r} / \frac{\mathrm{d} \ln n}{\mathrm{~d} r}>\frac{2}{3}$

that is a condition most commonly verified in well confined plasma experiments, the thermal energy flux is outward and the particle flux is inward. We note that the experimental evidence of the particle inflow process in well confined plasmas mentioned above has expanded over the years and by now it is well documented.

It has been suggested (by the referee) that the condition $-\mathrm{d} T_{\mathrm{e}} / \mathrm{d} z>-(2 / 3)\left(T_{\mathrm{e}} / \rho\right) \mathrm{d} \rho / \mathrm{d} z$ be compared to the instability condition of the classical Schwarzchild instability for stellar interiors $-\mathrm{d} T / \mathrm{d} z>g / C_{\mathrm{p}}$ where $g$ is the gravity acceleration and $C_{\mathrm{p}}$ is the heat capacity at constant pressure. In fact in both cases a transport of temperature associated with an opposing flow of matter is involved.

\section{Mode growth rates}

We refer to Eq. (39) and note that the quadratic form associated with it is

$\left(\frac{\gamma_{0} H_{0}}{v_{\mathrm{A}}^{2}}\right)^{2}\left\langle\left(1+\frac{4}{3} \frac{k^{2} v_{\mathrm{A}}^{2}}{k^{2} v_{\mathrm{A}}^{2}+\gamma_{0}^{2}}\right)\left(\tilde{\xi}_{z k}\right)^{2}\right\rangle=E_{k}^{0}\left\langle\left(\bar{k} \tilde{\xi}_{z k}\right)^{2}\right\rangle-\left\langle\left(\bar{k}^{2} \tilde{\xi}_{z k}\right)^{2}\right\rangle$

$+C_{0}^{0}\left\langle\left(\frac{\mathrm{d} \tilde{\xi}_{z k}}{\mathrm{~d} \bar{k}}\right)^{2}\right\rangle-\left\langle\left[\frac{\mathrm{d}}{\mathrm{d} \bar{k}}\left(\bar{k} \tilde{\xi}_{z k}\right)\right]^{2}\right\rangle$. 
In the realistic limit where $\gamma_{0}^{2}<k^{2} v_{\mathrm{A}}^{2}$ this form can be used as a variational principle to evaluate $\left(\gamma H / v_{\mathrm{A}}\right)^{2}$. Considering this limit the "ordering of maximum information" for which all the remaining terms in Eq. (52) are retained is $k^{4} \sim k_{0}^{2} / H_{0}^{2}=1 / \Delta_{0}^{4}$, $\gamma_{0}^{2} \sim v_{\mathrm{A}}^{2} / H_{0}^{2}, \epsilon_{k} \sim k^{2} / k_{0}^{2} \sim C_{0} / 3$.

$\Gamma_{0}^{2} \equiv \frac{7}{3}\left(\frac{\gamma_{0} H_{0}}{v_{\mathrm{A}}}\right)$

and obtain

$\left\{\Gamma_{0}^{2}-\bar{k}^{2}\left(E_{k}^{0}-\bar{k}^{2}\right)\right\} \tilde{\xi}_{z k}=\bar{k} \frac{\mathrm{d}^{2}}{\mathrm{~d} \bar{k}^{2}}\left(\tilde{\xi}_{z k} \bar{k}\right)-C_{0}^{0} \frac{\mathrm{d}^{2}}{\mathrm{~d} \bar{k}^{2}} \tilde{\xi}_{z k}$.

The lowest (even) eigensolution, Eq. (41), of this gives

$\Gamma_{0}^{2}=C_{0}^{0}, \quad E_{k}^{0}=3+C_{0}^{0}$.

It is clear that the instability requires relatively peaked temperature profiles, that is $\eta_{T}>2 / 3$ corresponding to the thermorotational instability. In particular,

$\gamma_{0}^{2}=\left(\frac{\sqrt{3} 6}{35}\right) \frac{v_{\mathrm{A}}}{H_{0}} \Omega_{k}\left(\eta_{T}-\frac{2}{3}\right), \quad \epsilon_{k}=\varepsilon_{0}+\frac{2}{5}\left(\eta_{T}-\frac{2}{3}\right)$

where $\varepsilon_{0} \equiv 1 /\left(k_{0} H_{0}\right)<1$.

In the case where $C_{0} \sim 1$ and $\epsilon_{k}>\varepsilon_{0}^{2 / 3}$ we find

$\Delta_{z} \simeq \Delta_{0}\left(\frac{3 \epsilon_{k}}{\left|C_{0}\right|}\right)^{1 / 4}$

and

$\gamma_{0} \simeq \sqrt{\frac{3}{7}}\left(\frac{v_{\mathrm{A}}}{H_{0}} \Omega_{k}\right)^{1 / 2}\left(\epsilon_{k}\left|C_{0}\right|\right)^{1 / 4}$

showing that the dependence of $\Delta_{z}$ and $\gamma_{0}$ on $\epsilon_{k}$ is weak, in this case.

The next (odd) eigenfunction, Eq. (47), corresponds to the ballooning mode investigated already by Coppi \& Keyes (2003). In this case the dispersion relation is $\Gamma_{0}^{2}=2+3 C_{0}^{0}$ while $E_{k}^{0}=$ $5+C_{0}^{0}$. Clearly the mode can be unstable even if $C_{0}^{0}<0$ that is for $\eta_{T}<2 / 3$ and

$\gamma_{0}^{2} \simeq \frac{6}{7} \frac{v_{\mathrm{A}}}{H_{0}}\left[\frac{\sqrt{3} 3}{5}\left(\eta_{T}-\frac{2}{3}\right) \Omega_{k}+\frac{v_{\mathrm{A}}}{H_{0}}\right]$.

We observe that, relative to the case investigated by Coppi \& Keyes (2003) the relevant growth rate is increased by the term $k_{0} H_{0}\left(\eta_{T}-2 / 3\right) 3 / 5$.

The next (even) "harmonic" has the form

$\tilde{\xi}_{z k}=\tilde{\xi}_{z 0}\left(1+\alpha_{k} \bar{k}^{2}\right) \exp \left(-\frac{\bar{k}^{2}}{2}\right)$,

where

$\alpha_{k}=\frac{1}{2}\left(1-\frac{\Gamma_{0}^{2}}{C_{0}^{0}}\right), \quad E_{k}^{0}=7+C_{0}^{0}$,

$\Gamma_{0}^{4}-6\left(1+C_{0}^{0}\right) \Gamma_{0}^{2}+C_{0}^{0}\left(14+5 C_{0}^{0}\right)=0$,

which allows the instability to exist up to $C_{0}^{0}=-14 / 5$. We note that when $C_{0}^{0} \rightarrow 0, \alpha_{k} \rightarrow-3 / C_{0}^{0}$ as $\Gamma_{0}^{2} \rightarrow 6$. When $C_{0}^{0}<-14 / 5$ a higher eigensolution has to be considered.

\section{Oscillatory modes}

A class of modes that are oscillatory and weakly damped can be found when $\eta_{T}$ is well below $2 / 3$, that is when $C_{0}$ is negative and finite. Clearly, these modes can be significant only if their amplitudes can be sustained by a non-linear coupling to modes that are self excited. Unlike the unstable modes discussed in the previous sections those considered here have $k_{R}^{2}>k_{0}^{2}$ and in particular $k_{R}^{2} \simeq k_{0}^{2}\left(1+\varepsilon^{k}\right)$ where $0<\varepsilon^{k}<1$. This means that the restoring effect of magnetic field line bending prevails over that of the radial gradient of the rotation frequency. Then instead of Eq. (39) we consider

$$
\begin{aligned}
& \left\{-\left(\frac{\omega_{o} H_{0}}{v_{\mathrm{A}}^{2}}\right)^{2}\left(\frac{7}{3}+\frac{4}{3} \frac{\omega_{0}^{2}}{k^{2} v_{\mathrm{A}}^{2}-\omega_{0}^{2}}\right)+k^{2} H_{0}^{2}\left(\varepsilon^{k}+\frac{k^{2}}{k_{0}^{2}}\right)\right\} \tilde{\xi}_{z k} \\
& \simeq k \frac{\mathrm{d}^{2}}{\mathrm{~d} k^{2}}\left(k \tilde{\xi}_{z k}\right)+\frac{\left|C_{0}\right|}{3} k_{0}^{2} \frac{\mathrm{d}^{2}}{\mathrm{~d} k^{2}} \tilde{\xi}_{z k} .
\end{aligned}
$$

Because of the singularity appearing in Eq. (61), $\omega_{0}$ will acquire a relatively small imaginary part, that is $\omega_{0}=\delta \omega_{0}+\mathrm{i} \gamma_{I}$. Therefore, it is convenient to refer to the quadratic form that can be obtained from Eq. (61), observing that $\tilde{\xi}_{z k}$ is complex in this case, in order to ascertain the sign of $\gamma_{I}$. This is

$$
\begin{aligned}
& \left.\left(\frac{\omega_{0} H_{0}}{v_{\mathrm{A}}^{2}}\right)^{2}\left\langle\frac{7}{3}+\frac{4}{3} \frac{\omega_{0}^{2}}{k^{2} v_{\mathrm{A}}^{2}-\omega_{0}^{2}}\right)\left|\tilde{\xi}_{z k}\right|^{2}\right\rangle \simeq \\
& H_{0}^{2}\left\langle k^{2}\left(\varepsilon^{k}+\frac{k^{2}}{k_{02}}\right)\left|\tilde{\xi}_{z k}\right|^{2}\right\rangle \\
& +\langle| \frac{\mathrm{d}}{\mathrm{d} k}\left(\left.k \tilde{\xi}_{z k}\right|^{2}\right\rangle+k_{0}^{2} \frac{C_{0} \mid}{3}\left\langle\left|\frac{\mathrm{d}}{\mathrm{d} k} \tilde{\xi}_{z k}\right|^{2}\right\rangle .
\end{aligned}
$$

It is also convenient to introduce dimensionless variables defining

$$
\begin{array}{ll}
\left(\frac{\omega_{0} H_{0}}{v_{\mathrm{A}}}\right)^{2} \equiv \frac{1}{7} k_{0}^{2} \Delta_{z}^{2}\left|C_{0}\right| \bar{\omega}^{2}, & \bar{E}^{k} \equiv \varepsilon^{k}\left(k_{0} \Delta_{z}\right)^{2} \\
\Delta_{z}^{6}=\frac{3}{\left|C_{0}\right|} \frac{H_{0}^{2}}{k_{0}^{4}}=\frac{3}{\left|C_{0}\right|} \frac{\Delta_{0}^{4}}{k_{0}^{2}}, & \Delta^{2} \equiv \frac{7}{\left|C_{0}\right|}\left(\frac{\Delta_{0}}{\Delta_{z}}\right)^{4} .
\end{array}
$$

Thus $1 / k_{0}<\Delta_{z}<\Delta_{0}, A^{2}>1$ and Eq. (61) becomes

$$
\left\{-\bar{\omega}^{2}\left(1+\frac{4}{7} \frac{\bar{\omega}^{2}}{\overline{\bar{k}}^{2} A^{2}-\bar{\omega}^{2}}\right)+\overline{\bar{k}}^{2}\left(\overline{\bar{k}}^{2}+\bar{E}^{k}\right)\right\} \tilde{\xi}_{z k} \simeq \frac{\mathrm{d}^{2}}{\mathrm{~d} \overline{\bar{k}}^{2}} \tilde{\xi}_{z k} .
$$

We note that, for $E^{k}>1$, Eq. $\left(61^{\prime}\right)$ gives

$\omega_{0}^{2} \simeq \frac{\sqrt{3}}{7}\left|C_{0}\right| \frac{\Delta_{z}^{2}}{\Delta_{0}^{2}} \frac{v_{\mathrm{A}}}{H_{0}} \Omega_{k} \bar{\omega}^{2}, \quad \bar{\omega}^{4} \simeq \bar{E}^{k}$.

The fact that $|\operatorname{Im} \bar{\omega}|<|\operatorname{Re} \bar{\omega}|$ is related to $A^{2}$ being larger than unity. In particular, $\gamma_{I}<0$. The case where $\eta_{T}>2 / 3$ and $\epsilon^{k}>0$ remains to be analyzed.

\section{Tridimensional, tightly wound spirals}

Referring to Eq. (13), we consider modes that are represented by $\hat{v}_{\phi}=\tilde{v}_{\phi}\left(R-R_{0}, z\right)$

$$
\exp \left\{\gamma_{0} t+\mathrm{i}\left[k_{R}\left(R-R_{0}\right)+m_{\phi}\left(\phi-\Omega_{o} t\right)-\delta \omega_{0} t\right]\right\}
$$


where $\Omega_{0} \equiv \Omega_{k}\left(R_{0}\right),\left|k_{R}\right| \simeq k_{0}, \tilde{\mathrm{v}}_{\phi}\left(R-R_{0}, z\right)$ varies over a radial scale distance that is considerably larger than $L_{c} \equiv 1 / k_{0}$ and we take $m_{\phi}$ to be relatively low. These modes are of the spiral type and we discuss at first the case where $\delta \omega_{0}=0$ and $\gamma_{0}$ is given approximately by the dispersion relations for axisymmetric modes analyzed in Sect. 4, for

$\gamma_{0}^{2}>m_{\phi}^{2}\left(\frac{\mathrm{d} \Omega}{\mathrm{d} R}\right)^{2} \Delta_{R}^{2} \sim m_{\phi}^{2} \Omega^{2}\left(\frac{\Delta_{R}^{2}}{R^{2}}\right)$,

where $\Delta_{R}$ is the radial width over which the mode is localized. In fact, this is a rather easy condition to satisfy as $\gamma_{0} \gtrsim v_{\mathrm{A}} / H_{0}$, and $v_{\mathrm{A}}^{2} / H_{0}^{2}>m_{\phi}^{2} \Omega_{k}^{2}\left(\Delta_{R} / R\right)^{2}$ for $\beta>m_{\phi}^{2}\left(\Delta_{R} / R\right)^{2}$ where $\beta \sim c_{s}^{2} / v_{\mathrm{A}}^{2}, c_{\mathrm{s}}$ is the sound velocity and $H_{0} \Omega_{k} \sim c_{\mathrm{s}}$.

We observe that the low- $m_{\phi}$ spiral modes considered here are directly connected to the axisymmetric modes discussed in Sects. 5 and 7. Referring to the radial displacement $\hat{\xi}_{R}$ we note that its expression, consistent with that of $\hat{v}_{\phi}$ given by Eq. (65), can be expanded as follows

$\hat{\xi}_{R} \simeq \exp \left[\mathrm{i} k_{R}\left(R-R_{0}\right)-\mathrm{i} m_{\phi}\left(\Omega_{k} t-\phi\right)+\gamma_{0} t\right]$

$\times\left[F_{0}\left(R-R_{0}\right) \tilde{\xi}_{R 0}(z)+\tilde{\xi}_{R 1}\left(z, R-R_{0}\right)+\tilde{\xi}_{R}^{1}\left(z, R-R_{0}\right)\right]$

$\equiv \hat{\xi}_{R 0}+\hat{\xi}_{R 1}+\hat{\xi}_{R}^{1}$

where $k_{R}=k_{0}+\delta k_{R},-1<\delta k_{R} / k_{0}<0$ and

$\frac{\mathrm{d} F_{0}}{\mathrm{~d} R} \sim \frac{F_{0}}{\Delta_{R}},\left|\frac{\hat{\xi}_{R 1}}{\hat{\xi}_{R 0}}\right| \sim \frac{1}{\Delta_{R}\left|\delta k_{R}\right|}<1,\left|\frac{\hat{\xi}_{R}^{1}}{\hat{\xi}_{R 0}}\right| \sim \frac{1}{\Delta_{R} k_{0}}<1$.

The expansion (67) implies that we take

$\frac{1}{\Delta_{R} k_{0}}>\frac{1}{\left(\Delta_{R} \delta k_{R}\right)^{2}}$,

i.e. $\left|\delta k_{R} / k_{0}\right|>1 /\left|\Delta_{R} k_{0}\right|^{1 / 2}$, and we consider the case where $\tilde{\xi}_{R 1}$ and $\tilde{\xi}_{R}^{1}$ both are separable function of $z$ and $R-R_{0}$.

Then we refer to Eq. (31) and observe that when condition (66) is satisfied it reduces to

$$
\begin{aligned}
& {\left[\frac{\partial^{2}}{\partial z^{2}}\left(2 \Omega_{k} \frac{\mathrm{d} \Omega_{k}}{\mathrm{~d} R}\right)\left(1-\frac{z^{2}}{H_{0}^{2}}\right) \hat{\xi}_{R}-v_{\mathrm{A}}^{2}\left(\frac{\partial^{2}}{\partial R^{2}}+\frac{\partial^{2}}{\partial z^{2}}\right) \hat{\xi}_{R}\right]} \\
& -\Omega_{k}^{2} \frac{\partial^{2}}{\partial R \partial z}\left(z \frac{\hat{\rho}}{\rho_{0}}\right)+2 \gamma_{0} \frac{\mathrm{d}(\Delta \gamma)}{\mathrm{d} R} \frac{\partial}{\partial R} \hat{\xi}_{R} \\
& \simeq\left[\gamma_{0}^{2}+\mathrm{i} 2 \gamma_{0}(\Delta \gamma)\right]\left[\frac{4}{3} k_{0}^{2} \hat{\xi}_{R}-\left(\frac{\partial^{2}}{\partial R^{2}}+\frac{\partial^{2}}{\partial z^{2}}\right) \hat{\xi}_{R}\right],
\end{aligned}
$$

where $\gamma_{t} \equiv \gamma_{0}+\mathrm{i} m_{\phi}\left(\mathrm{d} \Omega_{k} / \mathrm{d} R\right)\left(R-R_{0}\right) \equiv \gamma_{0}+\mathrm{i}(\Delta \gamma), \hat{\rho} / \rho_{0} \simeq$ $C_{0} z \tilde{\xi}_{z} / H_{0}^{2}$, and $\partial \hat{\xi}_{z} / \partial z \simeq-\partial \hat{\xi}_{R} / \partial R$. We notice that all the terms proportional to $\left(R-R_{0}\right)$ are grouped on the r.h.s. of Eq. (69) and that, for $k_{R}^{2} \gg \partial^{2} / \partial z^{2}$, this reduces to

$$
\begin{aligned}
& \frac{7}{3} \gamma_{0}^{2} k_{0}^{2} \hat{\xi}_{R}+i 2(\Delta \gamma) \gamma_{0} \frac{7}{3} k_{0}^{2} \hat{\xi}_{R 0} \\
& -\mathrm{i} 2 k_{0} \frac{\mathrm{d} F_{0}}{\mathrm{~d} R} \frac{\gamma_{0}^{2}}{F_{0}} \hat{\xi}_{R 0}-2 \gamma_{0}^{2} k_{R}\left|\delta k_{R}\right| \hat{\xi}_{R 0}-\gamma_{0}^{2} \frac{\partial^{2}}{\partial z^{2}} \hat{\xi}_{R 0} .
\end{aligned}
$$

We observe also that the slow dependence of $\hat{\xi}_{R}$ on $\left(R-R_{0}\right)$ can be expressed through $F_{0}\left(R-R_{0}\right)$ if

$\frac{1}{F_{0}} \frac{\mathrm{d} F_{0}}{\mathrm{~d} R}=\frac{\Delta \gamma}{\gamma_{0}} \frac{7}{3} k_{0}$ that gives

$$
F_{0} \simeq \exp \left[-\frac{7}{6} m_{\phi} k_{R} \frac{\Omega_{k}^{\prime}}{\gamma_{0}}\left(R-R_{0}\right)^{2}\right] \text {. }
$$

It is important to point out that, in order that $F_{0}$ be a localized function of $\left(R-R_{0}\right)$ the sign of $k_{R}$ and $m_{\phi}$ have to be related by the requirement that

$m_{\phi} k_{R} \Omega^{\prime}<0$

and, since $\Omega^{\prime}<0, m_{\phi} k_{R}>0$, this corresponds to trailing spirals.

Then we define

$\Delta_{R} \equiv\left|\frac{6}{7} \frac{\gamma_{0}}{\Omega^{\prime} m_{\phi} k_{R}}\right|^{1 / 2} \sim\left(\frac{\gamma_{0}}{\Omega_{k}} \frac{R_{0}}{k_{0} m_{\phi}}\right)^{1 / 2}$

and condition (66) becomes, approximately,

$\frac{m_{\phi}}{k_{0} R_{0}}<\frac{\gamma_{0}}{\Omega_{k}}<1$

with $\Delta_{R} k_{0} \sim\left(\gamma_{0} k_{0} R_{0}\right)^{1 / 2} /\left(\Omega_{k} m_{\phi}\right)^{1 / 2} \gg 1$. The condition $\left|\delta k_{R} / k_{0}\right|>1 /\left|\Delta_{R} k_{0}\right|^{1 / 2}$ can be easily satisfied as well for $\gamma_{0} \sim$ $\mathrm{v}_{A} / H_{0}, \Delta_{R} \sim\left(1 / k_{0}\right)\left[R_{0} /\left(m_{\phi} H_{0}\right)\right]^{1 / 2}$. In particular, taking into account that the vertical profiles of the modes represented by Eq. (67) are given by the theory of the corresponding axisymmetric $\left(m_{\phi}=0\right)$ modes, the spiral modes we consider become the form

$$
\begin{aligned}
& \hat{\xi}_{z} \simeq \tilde{\xi}_{z}^{0} \exp \left[-\frac{(R-R)^{2}}{\Delta_{R}^{2}}-\frac{z^{2}}{2 \Delta_{z}^{2}}\right] \\
& \times G_{0}^{0}(z) \sin \left\{k_{R}\left(R-R_{0}\right)-m_{\phi}\left[\Omega\left(R_{0}\right) t-\phi\right]\right\} \exp \left(\gamma_{0} t\right)
\end{aligned}
$$

where $G_{0}^{0}(z) \exp \left[-z^{2} /\left(2 \Delta_{z}^{2}\right)\right]$ represents the eigensolutions of Eq. (31).

We note that the "vertical ballooning" structure of the considered modes which depends on the density and temperature profiles of the equilibrium configuration, involves an analytical description that may be considered intermediate between a global and a shearing-box type of analysis (Umurhan 2008). It is evident from the derivation of the growth rates given in Sect. 7 that the radial gradient of the rotation frequency is the key factor for the excitation of the considered modes. In addition, a moderately peaked temperature profile corresponding to $\eta_{T}>2 / 3$ enhances the instability found when $\eta_{T}=2 / 3$ and add new unstable modes all of which require the radial gradient of the rotation frequency.

Finally, we may argue that, given the radial localization acquired by spiral modes, these should achieve higher amplitudes than axisymmetric modes which can be standing over considerably larger scale distances. Clearly, modes with higher values of $m_{\phi}$ have narrower radial profiles. Unlike axisymmetric modes, spiral modes have angular momentum (Bertin 2000). We note also that the radial localization distance $\Delta_{R}$ for spiral modes is related to the vertical pressure gradient and to the rotation frequency through the growth rate $\gamma_{0}$. This constitutes an intrinsic difference from the spiral modes found for galactic disks whose features are not considered to depend on the characteristics of their vertical profiles.

A first report of the theory of these spiral modes and of those analyzed in the next section was given by Coppi (2008b). The excitation of modes of this kind in the proximity of a black hole has been proposed as an explanation of high frequency quasiperiodic-oscillations (QPO's) by Coppi \& Rebusco (2008a). 


\section{Convective spirals}

Another kind of spirals is of special interest in view of the role they can have in the outward transport of angular momentum as needed for mass accretion by the central object. These spiral are oscillatory, in the frame where they corotate with the plasma at a given radius $R=R_{0}$, and are of the convective type, radially, over scale distances that can be considerably larger than the "microscopic" scale distances such as $\Delta_{0}$ that have been introduced. In fact, they may characterize the "equilibrium" state of a large class of accretion disks providing a means for angular momentum flow away from the central object.

Clearly, the modes here are derived from the axisymmetric oscillatory modes discussed in Sect. 8. The vertical structure of these modes can be given by the lowest order solution of Eq. (61), that is

$\hat{\xi}_{z} \propto G_{0}(z)=\exp \left(-\frac{z^{2}}{2 \Delta_{z}^{2}}\right)$,

when $\eta_{T}<2 / 3$ corresponding to relatively flat temperature profiles. The complete form of this mode can be represented as

$$
\begin{aligned}
& \hat{\xi}_{z} \simeq \tilde{\xi}_{z}^{0} \exp \left[-\frac{z^{2}}{2 \Delta_{z}^{2}}\right] \\
& \times \exp \left\{\mathrm{i}\left[m_{\phi}\left(\phi-\Omega_{k} t\right)+k_{R}\left(R-R_{0}\right)\right]\right. \\
& \left.-\mathrm{i}\left[\left(\delta \omega_{0}\right) t-\frac{\sigma_{R}}{2}\left(R-R_{0}\right)^{2}\right]\right\}
\end{aligned}
$$

over scale distances such that

$\left|\delta \omega_{0}\right|>\left|\left(R-R_{0}\right) \frac{\mathrm{d} \Omega_{k}}{\mathrm{~d} R}\right|$

corresponding to

$$
\left|R-R_{0}\right|<R_{0} \frac{1}{\beta^{1 / 6}}
$$

where $\beta=8 \pi p_{0} / B^{2}, k_{R}^{2} \simeq k_{0}^{2}$ and $\delta \omega_{0}$ can be derived from Eq. (61) after replacing $\omega_{0}$ by $\delta \omega_{0}$.

Clearly, this mode is oscillatory (in R) over shorter scale distances than that required by condition (79) as

$\sigma_{R} \simeq \frac{7}{3} \frac{m_{\phi} k_{R}}{\delta \omega_{0}} \frac{\mathrm{d} \Omega}{\mathrm{d} R}$.

Convective modes localized over shorter scale distances can be constructed out of this class of modes providing a means to transport energy and angular momentum associated with the mode away from $R_{0}$, provided $m_{\phi} \Omega^{\prime} k_{R}<0$. Clearly, this indicates a trailing spiral configuration as in the case of localized modes.

In particular, the type of mode packet centered around $\sigma_{R}=$ $\sigma_{0}$, that can be obtained by a superposition of the modes represented by Eq. (78) is exemplified by the integral

$$
\begin{aligned}
& \mathfrak{J} \equiv \Delta_{p}^{2} \int_{-\infty}^{+\infty} \mathrm{d} \sigma_{R} \\
& \times \exp \left[-\left(\sigma_{R}-\sigma_{0}\right)^{2} \Delta_{p}^{4}+\mathrm{i} \frac{\sigma_{R}}{2}\left(R-R_{0}\right)^{2}-\mathrm{i} \delta \omega_{0}\left(\sigma_{R}\right) t\right]
\end{aligned}
$$

where $\sigma_{0} \Delta_{p}^{2}>1$. Then, for

$$
\begin{aligned}
\delta \omega_{0}\left(\sigma_{R}\right) & \simeq \delta \omega_{0}\left(\sigma_{0}\right)+\left(\sigma_{R}-\sigma_{0}\right) \frac{\partial \delta \omega_{0}}{\partial \sigma_{R}} \\
+ & \frac{1}{2}\left(\sigma_{R}-\sigma_{0}\right)^{2} \frac{\partial^{2} \delta \omega_{0}}{\partial \sigma_{R}^{2}}
\end{aligned}
$$

we have

$$
\begin{aligned}
\mathfrak{I} \simeq & \sqrt{\pi} \exp \left\{\mathrm{i}\left[\frac{\sigma_{0}}{2}\left(R-R_{0}\right)^{2}-\delta \omega_{0}\left(\sigma_{0}\right) t\right]\right. \\
& \left.-\frac{1}{4 \Delta_{*}^{4}}\left[\frac{1}{2}\left(R-R_{0}\right)^{2}-\frac{\partial \delta \omega_{0}}{\partial \sigma_{R}} t\right]^{2}\right\}
\end{aligned}
$$

where

$\Delta_{*}^{4} \equiv \Delta_{p}^{4}+\frac{\mathrm{i}}{2} \frac{\partial^{2} \delta \omega_{0}}{\partial \sigma_{R}^{2}} t$

Therefore, we may define an effective transport coefficient representing the "convectivity" of the considered modes that is defined as

$D_{\text {eff }}=\frac{\partial}{\partial \sigma_{R}}\left(\delta \omega_{0}\right)=-\frac{3}{7} \frac{\left(\delta \omega_{0}\right)^{2}}{m_{\phi} k_{R} \Omega^{\prime}} \sim \frac{v_{\mathrm{A}}^{2}}{m_{\phi}} \frac{R_{0}}{\Omega_{k} H_{0}} \sim \frac{v_{\mathrm{A}} R_{0}}{m_{\phi}}\left(\frac{v_{\mathrm{A}}}{c_{s}}\right)$

considering that

$\delta \omega_{0} \simeq \frac{7}{3} \frac{m_{\phi} k_{R} \Omega^{\prime}}{\sigma_{R}}$

It is evident that $D_{\text {eff }}$ can have relatively large values and exceed the Shakura-Sunyaev coefficient (1973) for the outward transport of angular momentum, $D_{\mathrm{ss}} \sim \alpha_{\mathrm{ss}} c_{\mathrm{s}} H_{0}$, when $v_{\mathrm{A}}^{2}>$ $\alpha_{\mathrm{ss}} c_{\mathrm{s}}^{2}\left(H_{0}^{2} / R_{0}^{2}\right) m_{\phi}$

Then a reasonable scenario to be envisioned is one where the eigensolutions of Eq. (39) can be unstable and the corresponding (normal mode) spirals are radially localized around $R-R_{0}$. The amplification of convective modes can be tied to the coupling to linearly unstable modes providing a natural saturation process for the latter kind of mode. Moreover, we may argue that the minimum radius $R=R_{0}$ from which a significant rate of outward angular momentum transport can be produced by the excited spirals could represent the radius around which the accreting plasma accumulates. Finally, we note that when $\eta_{T} \geq 2 / 3$ the vertical structure of oscillatory modes has to be properly analyzed.

\section{Conclusions}

The investigation of the spectrum of plasma modes that can be excited in disk configurations in the prevalent gravity of a central object has led to new findings that include:

a. The identification of tridimensional spiral modes for which the vertical structure (unlike the case of galactic spirals) plays a key role in their properties.

b The vertical transport of both particles and thermal energy, in opposing vertical directions, produced both by axisymmetric and non-axisymmetric (e.g. spiral) modes which can lead to an outflow of plasma from the disk structure.

c. The coexistence of both standing and convective spiral modes, providing a means to transport angular momentum radially with significant rates. These may be consistent with those needed to justify the rates of mass accretion inferred from the observed luminosities of significant astrophysical objects.

We point out that the geometry of the disk structures from which plasma modes can be excited has a determining influence on the physical nature of these modes. Therefore theories adapted 
from ones concerning, for instance, cylindrical, plane, or homogeneous plasmas cannot be applicable necessarily to astrophysical disk structures. In fact, the importance of geometry has been made particularly evident by the kind of collective modes that have been observed and systematically investigated in magnetically confined laboratory plasmas.

Clearly, the analysis presented here needs to be extended by appropriate computational methods, beyond the limits of the quasi-linear approximation used in Sect. 6 in order to investigate the transport processes associated with the considered modes. For this however, the shortcomings of currently adopted computational approaches such as those pointed out by Regev \& Umurhan (2008) need to be resolved.

\footnotetext{
Acknowledgements. It is a pleasure to thank C. Crabtree and P. Rebusco for their comments and contributions to different subjects and issues dealt with in this paper and the (anonymous) referee for his suggestions . This work was sponsored in part by the US Department of Energy.
}

\section{References}

Balbus, S. A., \& Hawley, J. F. 1991, ApJ, 376, 214

Bertin, G. 2000, Dynamics of Galaxies (Publ. Cambridge Un. Press)

Blandford, R. D. 1976, MNRAS, 176, 465

Chandrasekhar, S. 1960, Proc. Natl. Acad. Sci., 46, 253

Coppi, B. 2008a, Europhys. Lett., 82, 19001

Coppi, B. 2008b, Paper P1 (E.P.S. Conference on Plasma Physics, Crete, Greece), 177

Coppi, B., \& Spight, C. 1978, Phys. Rev. Lett., 41, 551

Coppi, B., \& Coppi, P. S. 2001, Phys. Rev. Lett., 87, 051101

Coppi, B., \& Keyes, E. A. 2003, ApJ, 595, 1000

Coppi, B., \& Rousseau, F. 2006, ApJ, 641, 458

Coppi, B., \& Rebusco, P. 2008, P., Paper P5 (E.P.S. Conference on Plasma Physics, Crete, Greece), 154

Elvis, M. 2000, ApJ, 545, 689

Lovelace, R. V. E. 1976, Nature, 262, 649

Pringle, J. E. 1981, ARA\&A, 19, 137

Regev, O., \& Umurhan, O. H. 2008, A\&A, 481, 1

Shakura, N. I., \& Sunyaev, R. A. 1973, A\&A, 24, 337

Velikhov, E. P. 1959, Sov. Phys. JETP, 36, 995

Umurhan, O. H. 2008, A\&A 489, 953 